\title{
Using ProtoPNet for Interpretable Alzheimer's Disease Classification
}

\begin{abstract}
Sanaz Mohammadjafari ${ }^{\dagger, *}$, Mucahit Cevik ${ }^{\dagger}$, Mathusan Thanabalasingam $^{\dagger}$, Ayse Basar ${ }^{\dagger}$, for the Alzheimer's Disease Neuroimaging Initiative ${ }^{1}$

† Data Science Lab, Ryerson University, Toronto, Canada

Abstract

Early detection of Alzheimer's disease (AD) is significant for identifying of better treatment plans for the patients as the $\mathrm{AD}$ is not curable. On the other hand, lack of interpretability for the high performing prediction models might prevent incorporation of such models in clinical usage for AD detection. Accordingly, it is important to develop highly interpretable models which can create trust towards the prediction models by showing the factors that contribute to the models' decisions. In this paper, we use ProtoPNet architecture in combination with popular pretrained deep learning models to add interpretability to the AD classifications over MRI scans from ADNI and OASIS datasets. We find that the ProtoPNet model with DenseNet121 architecture can reach 90 percent accuracy while providing explanatory illustrations of the model's reasonings for the generated predictions. We also note that, in most cases, the performances of the ProtoPNet models are slightly inferior to their black-box counterparts, however, their ability to provide reasoning and transparency in the prediction generation process can contribute to higher adoption of the prediction models in clinical practice.
\end{abstract}

Keywords: Alzheimer disease, MRI image classification, Interpretability, ProtoPNet

\section{Introduction}

Alzheimer's Disease (AD) is the most common cause for dementia in elderly people [1]. It remains as one of the most prevalent incurable diseases, and its prevalence is only expected to increase. Nearly 50 million people have AD or related dementia worldwide [1]. In U.S. alone, 5.8 million people of age 65 or older have $\mathrm{AD}$, and this number is projected to increase to 13.8 million by 2050 . Furthermore, costs associated with $\mathrm{AD}$ and dementia could be as high as $\$ 1.1$ trillion by 2050 in U.S. [1].

Early detection of $\mathrm{AD}$ can contribute to identifying better treatment plans and enhances the overall quality of life for the patients. Various machine learning models that were trained on varying datasets have been considered for detecting AD [2-4]. One particularly relevant research area in this regard is image classification. Researchers have created machine learning models that are able to use MRI images from the brain, and successfully classify whether or not an individual has AD. Previous studies reported that some of these image classification models are able to predict AD more effectively than human experts [5].

Although these deep learning-based image classification models have become more and more accurate over time, similar to most neural network-based approaches, the intermediate processes within these models are not easily explainable to the humans. This implies that the models can effectively predict whether a given patient has AD or not. However, the reasoning of how a model reaches such conclusions is often difficult to understand [6]. Another complicating factor in adoption of these prediction models in practice is the difficulty in comparing results across studies due to inconsistent methods of using the data [7].

${ }^{1}$ Data used in preparation of this article were obtained from the Alzheimer's Disease Neuroimaging Initiative (ADNI) database (adni.Ioni.usc.edu). As such, the investigators within the ADNI contributed to the design and implementation of ADNI and/or provided data but did not participate in analysis or writing of this report. A complete listing of ADNI investigators can be found at:http://adni.loni.usc.edu/wp-content/ uploads/how_to_apply/ADNI_Acknowledgement_List.pdf.

*sanaz.mohammadjafari@ryerson.ca

This article is (C) 2021 by author(s) as listed above. The article is licensed under a Creative Commons Attribution (CC BY 4.0) International license (https://creativecommons.org/licenses/by/4.0/legalcode), except where otherwise indicated with respect to particular material included in the article. The article should be attributed to the author(s) identified above. 
Accordingly, lack of interpretability combined with difficulties in verifying and validating the research outcomes could lead practitioners not to accept findings of such studies.

Being able to validate the results of an experiment with sound reasoning of how the results were obtained is becoming more important as the theoretical models are brought to practice. In machine learning, this concept is often referred to as interpretability. Having an interpretable model introduces a measure that is simply assumed about in certain studies and but not necessarily verified; a trust in the results [8]. With models that show the reasoning of why certain inputs yield certain outputs, there is an elevated level of trust towards the results obtained by a model. Recent studies have incorporated the interpretability for AD detection to increase the prediction transparency of the underlying deep learning models. However, to the best of our knowledge, these studies mainly focus on creating meaningful representations of the input and use those to categorize input data instead of specifically incorporating interpretable methods $[9,10]$.

In this study, we consider interpretable image classification models for $\mathrm{AD}$ classification that have the ability to provide highly accurate predictions. The main objective of our study is to investigate models that are able to identify the key parts of an MRI image that result in $\mathrm{AD}$ prediction while also explaining how it comes to that particular prediction. We specifically include an interpretability layer called a prototypical part network (ProtoPNet) [11] in the models used for AD classification. This layer enables observing a model's reasoning for the predicted outcomes. We conduct a detailed numerical study to assess the effectiveness of the interpretability layer as well as the resulting prediction performance. We experiment with three different baseline models, namely, VGG16 [12], ResNet50 [13], DenseNet121 [14] and their enhanced versions with transfer learning and ProtoPNet layers.

The rest of this paper is organized as follows. Section 2 summarizes the recent studies on deep learning for $\mathrm{AD}$ classification and interpretability. Section 3 provides a brief overview the ProtoPNet architecture and deep learning models. In Section 4, we provide the results of our numerical study including summary statistics on model performances and illustrations that show the interpretability of the model predictions. The paper concludes with a discussion on key findings, study limitations, and future research directions in Section 5.

\section{Background}

Earlier studies on developing prediction models for AD detection focused on standard supervised learning methods such as SVMs and the variants $[15,16]$. Recent progress in deep learning methods for image classification as well as their automated feature extraction capabilities contributed greatly to usage of these approaches for $\mathrm{AD}$ classification $[2,3]$. For instance, Payan and Montana [4] used 3D convolutional neural networks (CNNs) and sparse autoencoders for $\mathrm{AD}$ detection and showed that 3D feature extraction considerable improved the performance of the prediction model.

While, the deep learning methods show a boost in AD prediction accuracy, training these complex models from scratch has a few drawbacks such as requiring extensive amounts of training data, heavy computational complexity and expensive parameter tuning process. The idea of transferring the extracted knowledge from a heavily trained deep learning model on a large dataset to a different domain can help alleviating these limitations [17].

Several studies have shown the transfer learning to be applicable and effective on MRI datasets $[18,19]$. Hon and Khan [18] implemented transfer learning on two popular CNNs, namely, VGG16 and Inception V4, for AD detection using OASIS MRI dataset [20]. Upon comparing the results of the models trained from scratch with the ones trained using transfer learning, they found that the accuracy score was significantly higher in the latter method, with the Inception model reaching an average accuracy score of $96.25 \%$ with transfer learning. In comparison, the VGG16 model that is trained from scratch was only able to achieve 
$74.12 \%$ accuracy. Transfer learning performed on MRI scans from the ADNI dataset using architectures such as VGGNet and ResNet [13] is also demonstrated to achieve better performance when compared to 3D-CNN model trained from scratch [21].

Although, with the help of transfer learning, deep learning models can achieve high performance for certain image classification tasks, the black-box nature of the neural networks may cause a lack of interpretability and transparency on how the model generates the predictions and associates the input features to the outputs [6]. A detailed survey on interpretability methods and the interpretability of the neural networks was provided in [22], where the interpretable machine learning methods were categorized into intrinsic methods - which employs a self explanatory structure such as decision trees and attention modelsand post-hoc methods - which employs a second model to explain the generated results. Prototypical Part Network (ProtoPNet) architecture was introduced in a recent study [11], which incorporates the comparison among image parts to learned prototypes in the network structure to provide both the prediction and the interpretation. The proposed model was evaluated on a dataset consisting of 200 bird species, and was able to generate a comparable accuracy score to non-interpretable models, while also preserving interpretability by explaining what parts of the bird images are used to generate the prediction.

The advances in the interpretability aspects of the deep learning has motivated a significant amount of research, especially in the medical domain. Recent works on AD detection have also incorporated the interpretability considerations. A novel deep learning model that consists of a CNN followed by a multi-layer perceptron was proposed in [9] to generate high resolution figures of $\mathrm{AD}$ probability map that can be used later for $\mathrm{AD}$ prediction, achieving an accuracy of $83.4 \%$ over the ADNI dataset. Additionally, a deep learning model developed in [10] was used to extract regional abnormality representations of the brain, and interpret and predict the $\mathrm{AD}$ progression based on the illustrations.

In this work, we develop different variants of the ProtoPNet model [11] to provide explanations for $\mathrm{AD}$ detection and highlight the brain regions affecting the prediction, as well as the similar prototypical images that shape the model's predictions. Our work is different from other studies that take into account the interpretability considerations due to the level of reasoning that ProtoPNet presents, which is different from post-hoc interpretability methods in various aspects. Firstly, using ProtoPNet, the process is built-in and interpretations are generated during the classification. Furthermore, it does not only provide a visualization to infer the procedure, but also provides the actual process of generating predictions. ProtoPNet also differs from intrinsic methods (e.g. attention-based approaches), since those methods only provide which part of image is signified without specific reasoning. On the other hand, ProtoPNet architecture provides the prototypical sample that model has found similarities with as the reason for the highlighted parts.

\section{Methodology}

In this section, we first provide details on the datasets used, as well as the preprocessing steps taken into account. Then, we review the structure of the prediction models and the ProtoPNet architecture. Finally, we provide details on the experimental setup including details on training and validation procedures.

\subsection{Dataset}

In our analysis, we use two different brain MRI scan datasets, namely, OASIS and ADNI datasets. We briefly summarize the characteristics of these datasets and the preprocessing steps below. 
- OASIS: This dataset consists of cross-sectional MRI scans from Open Access Series of Imaging Studies (OASIS-1 $)^{2}$ and contains data for two types of patients: AD and healthy patients [20]. There are 416 subjects in the dataset whose ages are between 18 and 96. The preprocessing steps are adopted from [18], which include choosing 100 random AD patients and another 100 random healthy patients. From each patient's MRI scan, 32 most informative axial plane slices are selected based on an entropy-based algorithm that creates 3,200 images for each group. The reason for this limited selection is to test the generalization capability of the transfer learning approach. The images are then normalized, and converted to RGB by stacking and resizing to $224 \times 224$ to fit the models' training configurations.

- ADNI: We collected the MRI scans from ADNI-1 ${ }^{3}$ screening data that is preprocessed with Multiplanar Reconstruction, Gradwarp and B1 correction. In total, we use $95 \mathrm{MRI}$ scans from patients with $\mathrm{AD}$ and $113 \mathrm{MRI}$ scans from healthy patients. We do not incorporate the demographic information in our analysis to be consistent with the OASIS dataset. We extract axial slices of MRI scans using a fast and accurate deep learning based skull stripping tool called DeepBrain [23]. Moreover, we normalize the slices and convert to RGB by stacking, and resizing the images to $224 \times 224$ to fit the models' training configurations. Samples of the axial slices are illustrated in Figure 1 for OASIS and ADNI dataset.

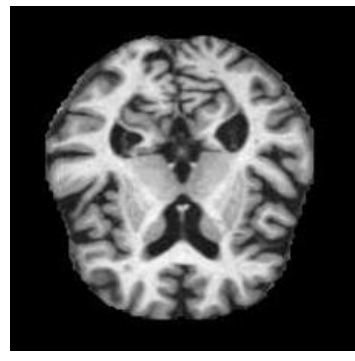

(a) OASIS dataset sample

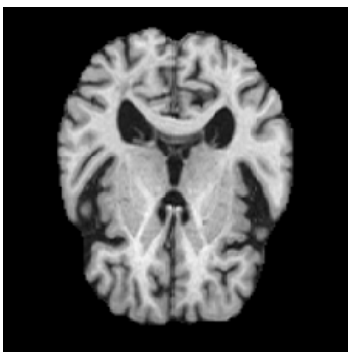

(b) ADNI dataset sample

Figure 1. Axial slices of MRI scans from OASIS and ADNI datasets

\subsection{Prediction Models}

We consider three different popular open source deep learning models with CNN structures for the AD classification task. These models include VGG16 [12], ResNet50 [13], DenseNet121 [14] that were originally created for the ImageNet Large Scale Visual Recognition Challenge (ILSVRC) [24] and were shown to perform well for AD classification [18, 21]. We briefly summarize the main characteristics of these models below.

- VGG16: A key feature of the VGG16 model is that, within each block, the number of filters increase by a factor of two, while the feature map size decreases by a factor of two. This allows for the model to differentiate between more basic features at the beginning, and form actual shapes as the inputs move deeper into the network. This model, with the exception of the output layer, strictly uses the 'ReLu' activation function at each layer. The model has $90.1 \%$ top-5 test accuracy in ImageNet. One drawback of the model is that it has $138 \mathrm{M}$ parameters to be learned, which typically leads to large training times depending on the hardware and the dataset size.

\footnotetext{
${ }^{2}$ Available at https://www .oasis-brains .org

${ }^{3}$ Available at adni.loni.usc.edu
} 
- ResNet50: This model introduced the idea of residual layers for image classification and won the ILSVRC in 2015. A residual layer has weights associated with layers deeper in the network to circumvent the vanishing gradient problem. ResNet50 has 48 convolutional layers, as well as one max pooling layer and one average pooling layer (i.e., a total of 50 layers). ResNet50 has $25 \mathrm{M}$ parameters which is considerably smaller than VGG16. The model has $92.1 \%$ top-5 test accuracy in ImageNet.

- DenseNet121: DenseNet is similar to ResNet, with some structural differences that make it more efficient than ResNet in terms of computational complexity and number of parameters (which is $8 \mathrm{M}$ ), while maintaining the same level of performance. In DenseNet, the input to the next layer is the concatenation of all the previous layers' outputs, rather than just the outputs of the last two layers as in ResNet. Each block is a group of layers, and the weights from each layer are sent to the concatenation step, rather than only the closest layer which makes it a desirable deep model for complex classification problems. DenseNet121 has 116 convolutional layers, along with one pooling layer, three transition layers and one classification layer. The model has $92.3 \%$ top-5 test accuracy in ImageNet.

\subsection{ProtoPNet Architecture}

The main idea behind ProtoPNet is to compare pieces of an image of an identified class to the pieces of images that are in the known classes. We adopt the ProtoPNet idea to train AD prediction models with the brain MRI scans. That is, the model is expected to compare parts of each MRI scan with a predetermined set of MRI scans namely prototypes, belonging to people with $\mathrm{AD}$ as well as to those that are healthy. The purpose of these prototypes is to describe the most relevant portions of MRI brain scans that match the prototype class; the number of prototypes that fall under AD would be defining traits in MRI scans of Alzheimer's patients, similar to how a neurologist would use key features to identify the disease. The patches from the CNN outputs represent a section of the full image, and we use these patches to compare with the prototypes. Note that, in [11], ProtoPNet architecture was tested on the problem of predicting more than 200 bird species using the models pretrained with ImageNet dataset. In the AD classification problem, there are only two classes (AD vs healthy), which potentially makes the underlying prediction task substantially easier.

The ProtoPNet structure illustrated in Figure 2 consists of three parts including a CNN part responsible for feature extraction with a structure selected from the prediction models discussed in Section 3.2. The second part is a prototype layer consisting of prototype units $g_{p_{j}}$ which compute the squared L2 distances between each prototype $p_{j}$ and all the patches of the image $x$ passed through the convolutional layers and invert the distances to create the similarity scores. These similarity scores define an activation map that shows the most similar parts of an image with the prototypes that is the reasoning behind class prediction. The similarity scores are reduced to a single value per prototype unit using global max pooling layer. The equation (3.1) shows the output of the prototype unit $g_{p_{j}}$ where $z=f(x)$ represents the patches of the image $x$ passed through the convolutional layers.

$$
g_{p_{j}}(z)=\max _{\tilde{z} \in \operatorname{patches}(z)} \log \frac{\left\|\tilde{z}-p_{j}\right\|_{2}^{2}+1}{\left\|\tilde{z}-p_{j}\right\|_{2}^{2}+\epsilon}
$$

Large values for the similarity scores indicate a small distance between the patches and the prototypes and thus, a similar structure between the training image and the prototypes. Finally, the third part is a fully connected layer with softmax activation function, which is trained to generate predicted probability of the class based on the similarity scores and the related weights. 


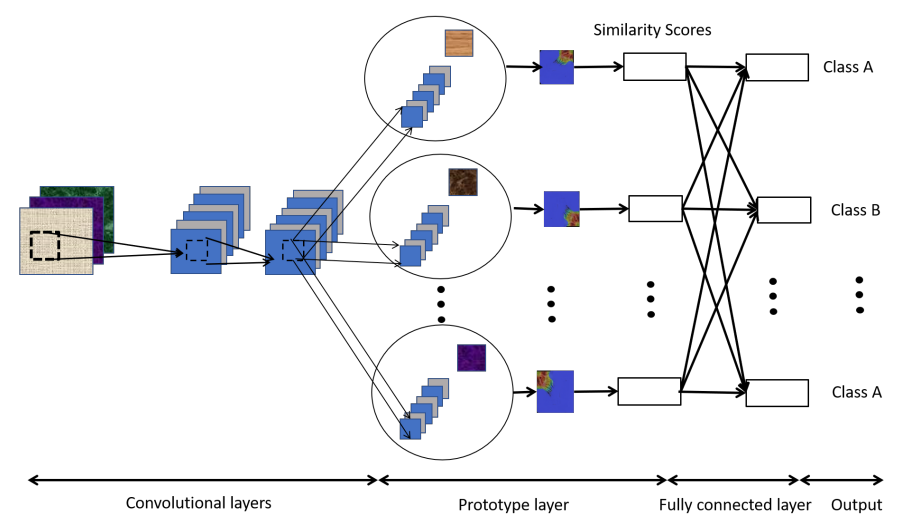

Figure 2. ProtoPNet architecture [11]

The training procedure of the model consists of three steps similar to the model's structure. In the first step, convolutional layers and prototype layer are trained to learn a latent space where important patches for classification are focused around the same class prototypes and the other patches from the opposite class prototypes are well separated. The training is based on the minimization of the cross entropy loss for misclassification, a cluster cost to encourage the patches to be similar to a prototypes of the same class and a separation cost to discourage them from opposite class prototypes. In the second step, the prototypes are projected onto the nearest training patches. In the final step, only the last fully connected layers are trained to tune the final weights for prediction where we aim for a weak connection between prototypes of opposite classes, and for the prediction to depend less on the negative reasoning.

\subsection{Experimental Setup}

We evaluate the model performances using the accuracy, precision and recall metrics. We use $k$-fold cross validation with $k=5$ to validate the models. Furthermore, each experiment is repeated five times to remove random initialization effect. We compare the performances of the three baseline deep learning models against their transfer learning-enhanced versions (i.e., using weights from the pretrained models in ImageNet dataset) as well as against the versions that includes ProtoPNet layers. For the transfer learning enhanced models, the deep learning models are used for feature extraction and after the addition of two dense layers to get the output, training is done on the last fully connected layers. We also performed hyperparameter tuning over different combinations of optimizers, learning rates and dropout rates. The final hyperparameter values for each model is reported in Table 1.

\begin{tabular}{|c|c|c|c|}
\hline Model & Optimizer & Learning rate & Dropout rate \\
\hline VGG16 & RMSprop & $1 \mathrm{e}-3$ & 0.5 \\
\hline ResNet50 & RMSprop & $1 \mathrm{e}-5$ & 0.2 \\
\hline DenseNet121 & RMSprop & $1 \mathrm{e}-6$ & 0.2 \\
\hline
\end{tabular}

For the ProtoPNet models, 25 images are selected randomly from each class, creating a 50 image prototype set for the model. The model is trained for 30 epochs, where the first 5 epochs are used for CNN training and the rest are used for prototype projection, computing similarity score and optimizing the last part of the network. 


\section{Numerical Results}

In this section, we present the findings from our detailed numerical study. We first compare the performance of the classification models using OASIS and ADNI datasets. Then, we provide results on the interpretability of the models' predictions, as well as the prediction performance on a test sample.

\subsection{Performance of the Classification Models}

The average accuracy, precision and recall metrics for the prediction models are reported in Table 2a and $2 \mathrm{~b}$ for OASIS and ADNI datasets, respectively. As expected, the baseline models with the transfer learning achieve better performance values compared to the baseline models. For instance, we observe that transfer learning has increased the accuracy for the VGG16 model from $73 \%$ to $91 \%$ for the OASIS dataset and from $75 \%$ to $88 \%$ for the ADNI dataset. Overall, the results highlight the ability of transfer learning in improving the performance of the deep learning models when datasets are small, affirming that the deep learning-based image classification models typically require a large amount of training data. We note a decrease in the performance values for the majority of the ProtoPNet models for both datasets, except for ResNet50 in ADNI dataset. The best performance is achieved by DenseNet121 with $\approx 94 \%$ accuracy, outperforming the VGG16 and ResNet50 models.

Table 2. Comparison of models' performances, averaged over 5 repeats (mean \pm std)

(a) OASIS dataset

\begin{tabular}{|c|c|c|c|c|}
\hline Model & Performance metric & Baseline model & $\begin{aligned} & \text { Baseline model } \\
+ & \text { transfer learning }\end{aligned}$ & $\begin{array}{l}\text { ProtoPNet model } \\
+ \text { transfer learning }\end{array}$ \\
\hline \multirow{3}{*}{ VGG16 } & Accuracy & $0.7388 \pm 0.0079$ & $0.9179 \pm 0.0074$ & $0.8215 \pm 0.0100$ \\
\hline & Precision & $0.7837 \pm 0.0284$ & $0.9053 \pm 0.0311$ & $0.8351 \pm 0.0325$ \\
\hline & Recall & $0.7943 \pm 0.0277$ & $0.8391 \pm 0.0408$ & $0.7937 \pm 0.0092$ \\
\hline \multirow{3}{*}{ ResNet50 } & Accuracy & $0.7579 \pm 0.0201$ & $0.8579 \pm 0.0117$ & $0.8398 \pm 0.0208$ \\
\hline & Precision & $0.7496 \pm 0.0145$ & $0.8785 \pm 0.0023$ & $0.8557 \pm 0.0307$ \\
\hline & Recall & $0.7492 \pm 0.0069$ & $0.8927 \pm 0.0072$ & $0.8518 \pm 0.0151$ \\
\hline \multirow{3}{*}{ DenseNet121 } & Accuracy & $0.7385 \pm 0.0101$ & $0.9483 \pm 0.0005$ & $0.8717 \pm 0.0132$ \\
\hline & Precision & $0.7054 \pm 0.0343$ & $0.9304 \pm 0.0359$ & $0.8669 \pm 0.0180$ \\
\hline & Recall & $0.7197 \pm 0.0349$ & $0.9266 \pm 0.0375$ & $0.8725 \pm 0.0151$ \\
\hline \multicolumn{5}{|c|}{ (b) ADNI dataset } \\
\hline Model & Performance metric & Baseline model & $\begin{aligned} & \text { Baseline model } \\
+ & \text { transfer learning }\end{aligned}$ & $\begin{array}{l}\text { ProtoPNet model } \\
+ \text { transfer learning }\end{array}$ \\
\hline \multirow{3}{*}{ VGG16 } & Accuracy & $0.7576 \pm 0.0305$ & $0.8850 \pm 0.0175$ & $0.7170 \pm 0.0301$ \\
\hline & Precision & $0.3409 \pm 0.0028$ & $0.9177 \pm 0.0441$ & $0.7247 \pm 0.0439$ \\
\hline & Recall & $0.7986 \pm 0.0109$ & $0.9053 \pm 0.0531$ & $0.9215 \pm 0.0102$ \\
\hline \multirow{3}{*}{ ResNet50 } & Accuracy & $0.7706 \pm 0.0236$ & $0.8388 \pm 0.0030$ & $0.9030 \pm 0.0431$ \\
\hline & Precision & $0.5713 \pm 0.0341$ & $0.7930 \pm 0.0079$ & $0.8716 \pm 0.0390$ \\
\hline & Recall & $0.7694 \pm 0.0025$ & $0.8660 \pm 0.0115$ & $0.9154 \pm 0.0078$ \\
\hline \multirow{3}{*}{ DenseNet121 } & Accuracy & $0.7323 \pm 0.0022$ & $0.9475 \pm 0.0015$ & $0.9102 \pm 0.0253$ \\
\hline & Precision & $0.4896 \pm 0.0141$ & $0.9574 \pm 0.0020$ & $0.8457 \pm 0.0241$ \\
\hline & Recall & $0.6897 \pm 0.0019$ & $0.9670 \pm 0.0015$ & $0.8708 \pm 0.0317$ \\
\hline
\end{tabular}

\subsection{Interpreting Model Predictions}

We pass a set of prototypical images consisting of 25 images per class to the ProtoPNet model for comparison and similarity score calculation. The ProtoPNet model compares each extracted feature of the input image with different patches of these prototypical images and returns a similarity score as well as an activation map. This process is illustrated in 
Table 3 and 4 for a random healthy patient sample prediction procedure, explaining the comparison steps before the prediction. Each table consists of the actual image with the activated region marked inside a rectangle, the activation map of the original test image, the prototype patch part, the prototypical image and its activation map. The tables also include numerical metrics such as similarity score and the class connection which stands for the fully connected layer weights, and the multiplication of these two metrics called contributed points.

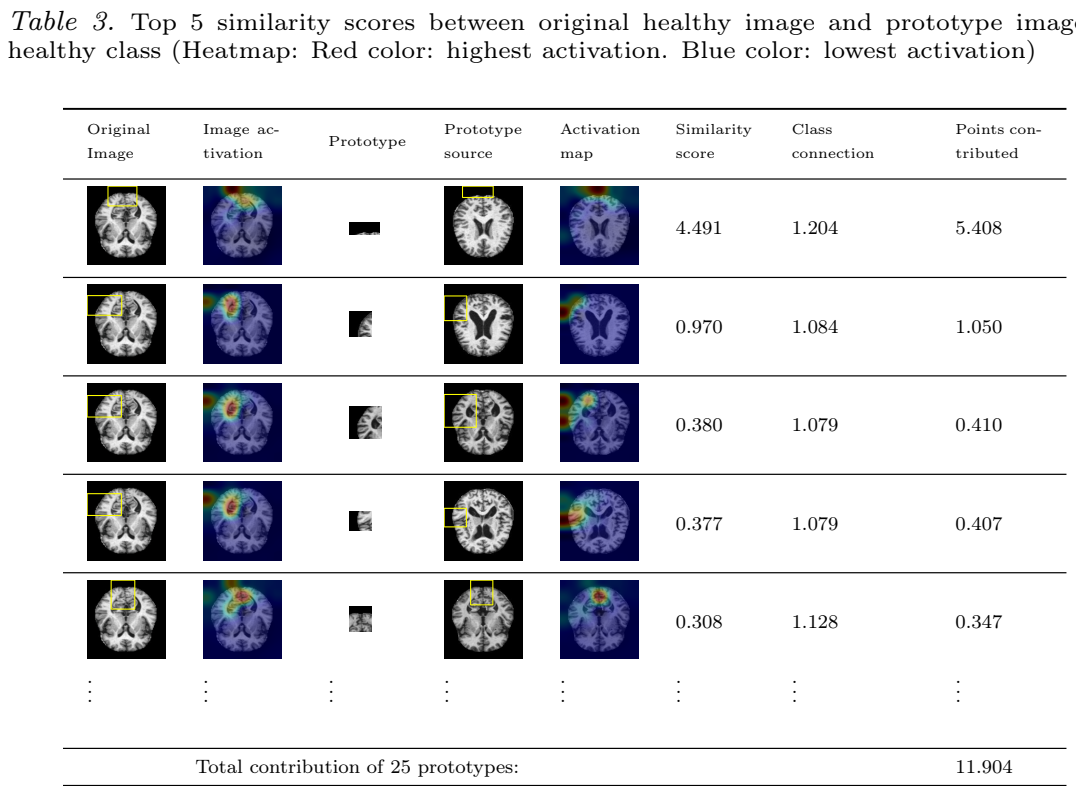

Table 4. Top 5 similarity scores between original healthy image and prototype images of AD class (Heatmap: Red color: highest activation. Blue color: lowest activation)

\begin{tabular}{llllllll}
\hline $\begin{array}{l}\text { Original } \\
\text { Image }\end{array}$ & $\begin{array}{l}\text { Image ac- } \\
\text { tivation }\end{array}$ & Prototype & $\begin{array}{l}\text { Prototype } \\
\text { source }\end{array}$ & $\begin{array}{l}\text { Activation } \\
\text { map }\end{array}$ & $\begin{array}{l}\text { Similarity } \\
\text { score }\end{array}$ & $\begin{array}{l}\text { Class } \\
\text { connection }\end{array}$ & $\begin{array}{l}\text { Points con- } \\
\text { tributed }\end{array}$ \\
\hline & & & & & & & \\
\hline
\end{tabular}

Table 3 shows the first 5 images out of 25 prototype images with the highest similarity scores that are selected from the healthy class along with the class connection to last layer and the points contributed. Table 4 reports the same metrics with the AD prototypes and finally the prediction is made based on the final total contribution which is 11.904 in 
healthy class and 2.339 in AD class, which leads to healthy class prediction because of major contribution from prototypical images, indicating a correct prediction made by the model. The illustrations in these tables depict the image activation map through a heat map that shows the most activated region by red color and the least activated region by blue color. We can also observe that same regions are activated between the two images as shown in the first sample of the Table 3. Specifically, the results show how the sample image has lower similarity scores with prototypes of the opposite class as well as low class connections where both of these metrics were designed to have lower values to encourage the model to focus on the positive reasoning, and similar prototypes for classification in the training process.

These tables including the images and the metrics illustrate the interpretability offered by ProtoPNet while clarifying the prediction procedure. The ProtoPNet model interpretability provides a part-level attention map which illustrates the regions used by the model in the prediction procedure. Moreover, model offers similar prototypical cases and the similarity between the target and these cases as rationale for the prediction procedure. It is important to note that other attention based models do not typically indicate the reason behind region selection for attention maps whereas, in the ProtoPNet case, the regions are selected based on similarity scores between the prototypical images and the test image. Compared to post-hoc interpretability methods, the ProtoPNet layer is a built-in intrinsic method which saves complexity and hyperparameter tuning cost of the second reasoning model as well as providing illustrations that are used in the prediction process rather than offering visualizations that do not explain the prediction generation process.

In order to provide a comparison of different models' performances, we demonstrated the reasoning procedure of one of the best and worst performing models by illustrating the highest similarity score achieved by a prototype for a random sample from AD class in Figure 3. Figure 3a shows the performance of a good performing DenseNet121 model that has $94 \%$ accuracy. The model is able to predict the class label correctly by finding a highly activated region from a prototype which belongs to the $\mathrm{AD}$ class as well. It is shown that the activated region in both the sample and the prototype represent the same regions of the axial slice. Figure 3b illustrates the prediction of high performing ResNet50 model which has $90 \%$ accuracy. We observe that the model is able to predict the image class correctly along with providing a clear illustration of activation map between the sample image and prototype image. On the other hand, Figure 3c shows the prediction for a poor performing VGG16 model (which has $72 \%$ overall prediction accuracy) over the same sample image. The model predicts the wrong class of healthy subject for the image based on prototype similarity scores and related weights. It is shown that the image have a high correlated activation map with a non brain region of the prototypical image. Accordingly, we note that high similarity score without the related weights does not guarantee a correct prediction.

\subsection{Impact of the Number of Prototypes}

Lastly, we investigate the impact of the number of prototypes on models' performance and reasoning using the best performing model, DenseNet121, on OASIS dataset by considering 25 and 100 prototypes per category. We observe that increasing the number of prototypes causes slight decrease in the model's performance (e.g., 2-3\% drop in accuracy, recall and precision values). The reasoning process of the model for a healthy test sample is illustrated in Table 5 and 6 . The mean total contribution over the number of prototypes has increased from $(0.476,0.0935)$ to $(0.807,0.376)$ for the same and opposite class from 25 to 100 prototypes. Previous studies do not mention the effect of the number of prototypes for model performance and interpretability, and in [11], it is noted that the prototypes ideally should be selected by domain experts to be representative of the dataset classes, or based 


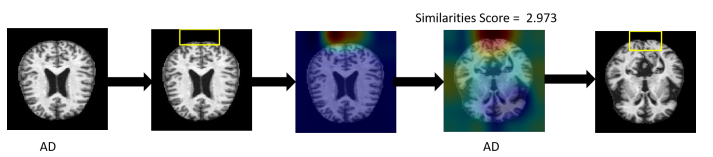

(a) DenseNet121-based ProtoPNet model

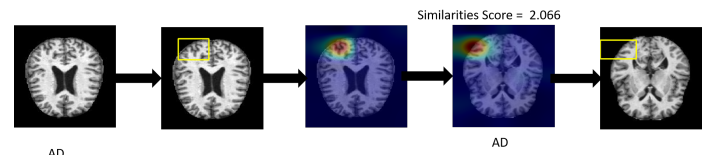

(b) ResNet50-based ProtoPNet model

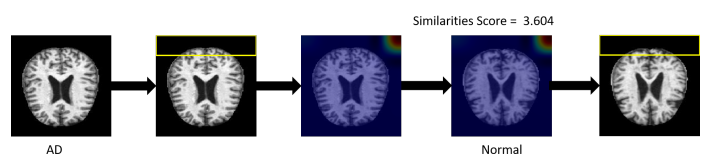

(c) VGG16-based ProtoPNet model

Figure 3. Top prototype samples from OASIS dataset (test image on the left)

on pruning methods in which the most similar prototypes that are activated by both classes should be excluded from the training procedure, since they do not present a unique feature.

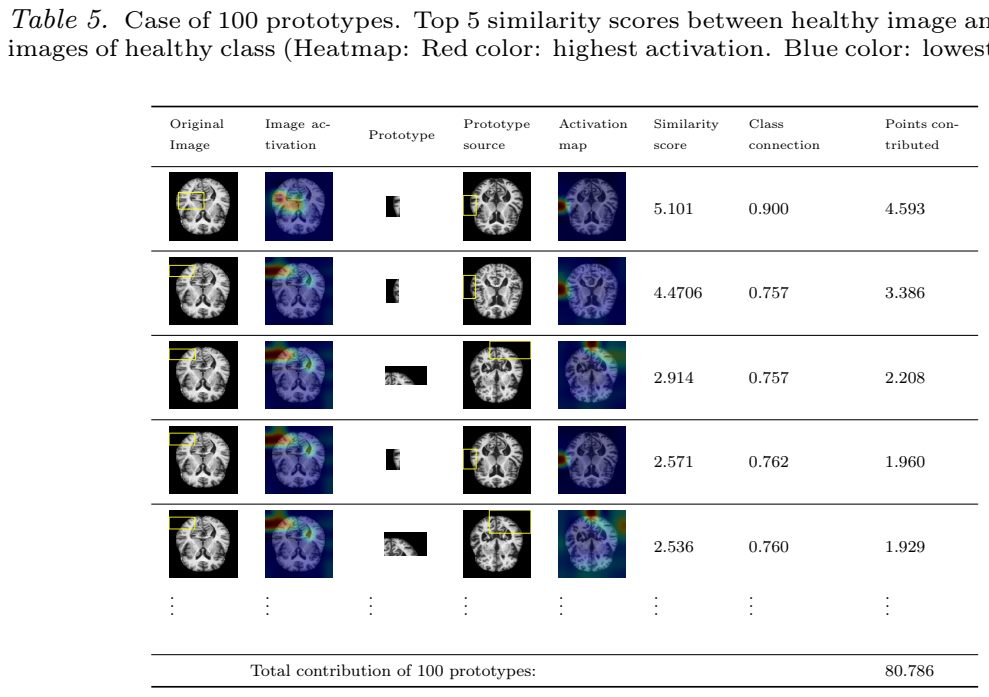

\section{Conclusion}

Fast progression of incurable Alzheimer's disease has signified the importance of reliable prediction methods to detect the disease in the early stages of its progress that can offer better medication options for the patients to control the symptoms and increase the patient's quality of life. Deep learning models can be particularly effective tools for AD detection due to their high accuracy and automated feature extraction ability, provided that they offer interpretability and transparency in prediction generation process to create certain level of trust towards these models. In this work, we evaluated the effectiveness of ProtoPNet architectures for detecting $\mathrm{AD}$, and assessed the provided interpretability from this architecture using brain MRI scans datasets. We used $k$-fold cross validation with $k=5$ along 
Table 6. Case of 100 prototypes. Top 5 similarity scores between healthy image and prototype images of AD class (Heatmap: Red color: highest activation. Blue color: lowest activation)

\begin{tabular}{llllllll}
\hline $\begin{array}{l}\text { Original } \\
\text { Image }\end{array}$ & $\begin{array}{l}\text { Image ac- } \\
\text { tivation }\end{array}$ & Prototype & $\begin{array}{l}\text { Prototype } \\
\text { source }\end{array}$ & $\begin{array}{l}\text { Activation } \\
\text { map }\end{array}$ & $\begin{array}{l}\text { Similarity } \\
\text { score }\end{array}$ & $\begin{array}{l}\text { Class } \\
\text { connection }\end{array}$ & $\begin{array}{l}\text { Points con- } \\
\text { tributed }\end{array}$ \\
\hline & & & & & & \\
\hline
\end{tabular}

with repeating the experiments five times and reporting the average performance values to remove any bias and random initialization effect. Our analysis with interpretable models opens future research avenues by providing a higher trust towards deep learning models for disease classification tasks where medical imaging is a significant tool for assessment and confirmation of the diseases such as cancer detection and diagnosis.

We recognize that different brain extraction tools along with various other preprocessing steps may potentially affect the results. We leave assessing the impact of these procedures on prediction model performance for future research. Another important future work would be to evaluate the interpretability and $\mathrm{AD}$ detection performance of the models on MRI scans of the patients over time and predict the progress of the disease from early to late stages. This problem contains more classes to predict and potentially leads to a more difficult prediction problem. In addition, segmentation of the brain and exploring the effect of different regions in the disease progression can be investigated as a future research direction.

\section{Acknowledgements}

Data collection and sharing for this project was funded by the Alzheimer's Disease Neuroimaging Initiative (ADNI) (National Institutes of Health Grant U01 AG024904) and DOD ADNI (Department of Defense award number W81XWH-12-2-0012). ADNI is funded by the National Institute on Aging, the National Institute of Biomedical Imaging and Bioengineering, and through generous contributions from the following: AbbVie, Alzheimer's Association; Alzheimer's Drug Discovery Foundation; Araclon Biotech; BioClinica, Inc.; Biogen; Bristol-Myers Squibb Company; CereSpir, Inc.; Cogstate; Eisai Inc.; Elan Pharmaceuticals, Inc.; Eli Lilly and Company; EuroImmun; F. Hoffmann-La Roche Ltd and its affiliated company Genentech, Inc.; Fujirebio; GE Healthcare; IXICO Ltd.; Janssen Alzheimer Immunotherapy Research \& Development, LLC.; Johnson \& Johnson Pharmaceutical Research \& Development LLC.; Lumosity; Lundbeck; Merck \& Co., Inc.; Meso Scale Diagnostics, LLC.; NeuroRx Research; Neurotrack Technologies; Novartis Pharmaceuticals Corporation; Pfizer Inc.; Piramal Imaging; Servier; Takeda Pharmaceutical Company; and Transition Therapeutics. The Canadian Institutes of Health Research is providing funds to support ADNI clinical sites in Canada. Private sector contributions are facilitated by the Foundation for the National Institutes of Health (www.fnih.org). The grantee organization is the Northern California Institute for Research and Education, and the study is 
coordinated by the Alzheimer's Therapeutic Research Institute at the University of Southern California. ADNI data are disseminated by the Laboratory for Neuro Imaging at the University of Southern California. The OASIS cross-sectional data were provided by PIs: D. Marcus, R, Buckner, J. Csernansky and J. Morris and supported by grants P50 AG05681, P01 AG03991, P01 AG026276, R01 AG021910, P20 MH071616 and U24 RR021382.

\section{References}

[1] Alzheimer's Association. Alzheimer's and Dementia Facts and Figures. Available from https://www.alz.org/alzheimers-dementia/facts-figures. Accessed on February 20, 2021. 2021.

[2] D. Shen, G. Wu, and H.-I. Suk. "Deep learning in medical image analysis". In: Annual review of biomedical engineering 19 (2017), pp. 221-248.

[3] H. I. Suk, S. W. Lee, D. Shen, et al. "Hierarchical feature representation and multimodal fusion with deep learning for AD/MCI diagnosis". In: NeuroImage 101 (2014), pp. 569-582.

[4] A. Payan and G. Montana. "Predicting Alzheimer's disease: a neuroimaging study with 3D convolutional neural networks". In: arXiv preprint arXiv:1502.02506 (2015).

[5] S. Klöppel, C. M. Stonnington, J. Barnes, et al. "Accuracy of dementia diagnosis - a direct comparison between radiologists and a computerized method". In: Brain 131.11 (2008), pp. 2969-2974.

[6] Q.-s. Zhang and S.-C. Zhu. "Visual interpretability for deep learning: a survey". In: Frontiers of Information Technology \& Electronic Engineering 19.1 (2018), pp. 27-39.

[7] Y. R. Fung, Z. Guan, R. Kumar, J. Y. Wu, and M. Fiterau. "Alzheimer's Disease Brain MRI Classification: Challenges and Insights". In: arXiv preprint arXiv:1906.04231 (2019).

[8] Z. C. Lipton. "The mythos of model interpretability". In: Queue 16.3 (2018), pp. 31-57.

[9] S. Qiu, P. S. Joshi, M. I. Miller, C. Xue, X. Zhou, C. Karjadi, G. H. Chang, A. S. Joshi, B. Dwyer, S. Zhu, et al. "Development and validation of an interpretable deep learning framework for Alzheimer's disease classification". In: Brain 143.6 (2020), pp. 1920-1933.

[10] E. Lee, J.-S. Choi, M. Kim, H.-I. Suk, et al. "Toward an interpretable Alzheimer's disease diagnostic model with regional abnormality representation via deep learning". In: NeuroImage 202 (2019), p. 116113 .

[11] C. Chen, O. Li, D. Tao, A. Barnett, C. Rudin, and J. K. Su. "This looks like that: deep learning for interpretable image recognition". In: NIPS. 2019, pp. 8930-8941.

[12] K. Simonyan and A. Zisserman. "Very deep convolutional networks for large-scale image recognition". In: arXiv preprint arXiv:1409.1556 (2014).

[13] K. He, X. Zhang, S. Ren, and J. Sun. "Deep residual learning for image recognition". In: Proceedings of the IEEE CVPR. 2016, pp. 770-778.

[14] G. Huang, Z. Liu, L. Van Der Maaten, and K. Q. Weinberger. "Densely connected convolutional networks". In: Proceedings of the IEEE CVPR. 2017, pp. 4700-4708.

[15] H. Bisgin, T. Bera, H. Ding, H. G. Semey, L. Wu, Z. Liu, A. E. Barnes, D. A. Langley, et al. "Comparing SVM and ANN based machine learning methods for species identification of food contaminating beetles". In: Scientific reports 8.1 (2018), pp. 1-12.

[16] S. Alam, G.-R. Kwon, and A. D. N. Initiative. "Alzheimer disease classification using KPCA, LDA, and multi-kernel learning SVM". In: IMA 27.2 (2017), pp. 133-143.

[17] J. Yosinski, J. Clune, Y. Bengio, and H. Lipson. "How transferable are features in deep neural networks?" In: arXiv preprint arXiv:1411.1792 (2014).

[18] M. Hon and N. M. Khan. "Towards Alzheimer's disease classification through transfer learning". In: 2017 IEEE BIBM. IEEE. 2017, pp. 1166-1169.

[19] N. Tajbakhsh, J. Y. Shin, S. R. Gurudu, R. T. Hurst, C. B. Kendall, M. B. Gotway, and J. Liang. "Convolutional neural networks for medical image analysis: Full training or fine tuning?" In: IEEE transactions on medical imaging 35.5 (2016), pp. 1299-1312.

[20] D. S. Marcus, T. H. Wang, J. Parker, J. G. Csernansky, J. C. Morris, and R. L. Buckner. "Open Access Series of Imaging Studies (OASIS): Cross-sectional MRI Data in Young, Middle Aged, Nondemented, and Demented Older Adults". In: Journal of Cognitive Neuroscience 19.9 (2007), pp. 1498-1507.

[21] A. Ebrahimi-Ghahnavieh, S. Luo, and R. Chiong. "Transfer Learning for Alzheimer's Disease Detection on MRI Images". In: 2019 IEEE IAICT. 2019, pp. 133-138.

[22] F. Fan, J. Xiong, and G. Wang. "On Interpretability of Artificial Neural Networks". In: arXiv e-prints (2020), arXiv-2001.

[23] I. Itzcovich. Deepbrain. https://github.com/iitzco/deepbrain. 2018.

[24] O. Russakovsky, J. Deng, H. Su, Krause, et al. "Imagenet large scale visual recognition challenge". In: International journal of computer vision 115.3 (2015), pp. 211-252. 